BULL. AUSTRAL. MATH. SOC.

VOL. $26(1982), 285-316$.

\title{
A MOLECULAR MODEL OF IONIC CONDUCTIVITY IN NERVE*
}

\author{
D.K. MCILROY \\ Communicated by James M. Hill
}

A mathematical model of the molecular processes responsible for the ionic conduction current across axonal membranes is proposed and analysed. It is shown that the model successfully predicts the various "instantaneous" current-voltage (IC-V) relationships of different kinds of axon and of axons in different bathing solutions: the criterion for the axon to have a linear IC-V is that the Debye length in the axonal membrane should be less than $d$, the distance between adjacent conducting pores. If $\lambda>d$ it is shown that the IC- $\mathrm{V}$ becomes nonlinear.

The model predicts that the axolemmic electric fields in the vicinity of the conducting pores are non-uniform and mappings of these fields are produced. The concepts of ionic selectivity and ionic permeability of the conducting ions are defined in terms of molecularly identifiable parameters of the model.

\section{Introduction}

Some nerve membranes have "instantaneously" (that is, on a time scale small compared with that of a nervous impulse) a linear current-voltage

Received 31 March 1982.

* This paper is based on an invited lecture given at the Australian Mathematical Society Applied Mathematics Conference held in Bundanoon, February 7-11, 1982. Other papers delivered at this Conference appear in Volumes 25 and 26. 
(IC-V) relationship, whilst others possess a nonlinear relationship. Thus, for example, the squid giant axonal membrane is linear in a natural ionic environment, but may become nonlinear when placed in an artificial bathing solution [11, 12]. On the other hand the axonal membrane of frog node of Ranvier is nonlinear even in a natural ionic environment [9]. In this article we propose a detailed molecular model of the ionic conduction process in nerve which encompasses all these phenomena.

In Section 2 we consider the structure of the axonal resting electric field in the light of the two classical limits of membrane theory, namely, the Planck limit and the Goldman limit, and we interpret the results of perfusion experiments in terms of these limits. However these interpretations must be strongly modified if our model of facilitated ionic transport through well-defined pores across the squid giant axonal membrane, which we propose in section 3, is valid. We show that this model leads directly to the Hodgkin-Huxley mathematical formulation of this axon's electrical properties; in addition we propose that the linearity of this IC-V relationship is due to the non-interaction of the ionic currents in adjacent pores. If however the Debye length $\lambda$ in the membrane is greater than the distance $d$ between adjacent conducting pores we propose, in section 4, that the resulting interaction of these ionic currents leads to a nonlinear relation for the IC-V which is the so called Goldman relationship. The latter is observed for some axonal membranes such as the frog node of Ranvier in a natural ionic environment.

Our theory enables us to deduce, in Section 5, the distribution of electric field in the vicinity of both sodium (Na) and potassium ( $K$ ) conducting pores of the squid giant axon. We are also able to deduce, in Section 6 , the transition of the IC-V relationship to nonlinearity when the squid giant axon is placed in choline bathing solutions. We propose that this nonlinearity however arises from the presence of impurity choline ions in the sodium conducting pores. The theory then leads us naturally to consider, in Section 7 , the effect of the presence of $\mathrm{Na}^{+}$in mainly potassium conducting pores and vice versa, which in turn leads us to define the concept of ionic selectivity of the two ionic conducting systems. Finally, in Section 8 , we define ionic permeability of an axonal membrane in terms of the model; using this definition we show that the determination of membrane permeability using radioactive tracer techniques is in 
complete agreement with the classical description of the resting axon.

\section{The structure of the axonal resting electric field}

Because of the large difference between the magnitudes of the axonal membrane conductances $g_{\mathrm{K}}$ and $g_{\mathrm{Na}}$ in the resting state as well as at the peak of an action potential, we can estimate the corresponding membrane potential differences as a first approximation from the Nernst equilibrium potentials for respectively $\mathrm{K}$ and $\mathrm{Na}$ ions alone. Higher approximations must take into account the simultaneous finite values of $g_{\mathrm{K}}$ and $g_{\mathrm{Na}}$, that is, of the non-equilibrium nature of the actual potential difference. But even if the calculated non-equilibrium corrections of the potential difference were small, or if present knowledge of the ionic diffusion coefficients were insufficient to evaluate the corrections accurately, the structure of the resting potential difference and its corresponding field would be of fundamental importance in the construction of molecular models of the mechanism responsible for the control of the nervous impulse because of the following observations.

The resting potential difference may be decreased by reducing the internal potassium concentration through perfusion of the axon. If the resting potential difference is substantially lowered by reducing only the internal potassium concentration under conditions of constant internal ionic strength action potentials cannot be initiated. But if the internal ionic strength is reduced in proportion to the potassium concentration action potentials can be initiated by depolarizations from resting potential differences in the range $30 \mathrm{mV}$ to zero (taking the outside of the axon as positive) [4], [17].

Now the average membrane field intensity $\bar{X}$ is related to the membrane potential difference $\phi$ and membrane thickness $\delta$ by

$$
\bar{X}=-\frac{\phi}{\delta}=\frac{1}{\delta} \int_{0}^{\delta} X(x) d x \text {, }
$$

and, if the measured potential difference is the actual potential difference across the membrane, becomes small as $\phi$ does. If $X(x)$ were uniform, $X(x)=\bar{X}$; action potentials could then be initiated by changes of a zow field (for example, at low internal ionic strength) and all 
microscopic mechanisms of the initiation of the nerve impulse which rely on the effects of the reduction of a high resting field would be inadequate. These include the wien effect on the dissociation of a weak electrolyte, the rotation of dipoles and the displacement of ions. But if, on the other hand, the resting field $X(x)$ is so distributed within the membrane that it remains high locally despite a low average value then the above highfield mechanisms remain tenable. The question is then: is the resting field uniform or distributed in the membrane?

To attempt to answer this question we first consider Planck's formulation of electrodiffusion as applied to axonal membranes. The resting membrane is in a steady (non-equilibrium) state, maintained in the long term by active transport. Since the membrane is thin as compared with the radius of the squid giant axon, we may regard it as an infinite plane region of dielectric constant $\varepsilon$ between $x=0$ (inner surface) and $x=\delta$ (outer surface) within which ionic species $j$ have concentrations $c_{j}(x)$, charges $z_{j} e^{\prime}$, and diffusion coefficients $D_{j}$, where $e^{\prime}>0$ is the elementary charge. The ions move through the membrane under the action of the electric field superimposed on diffusion. In the steady state the flux $F_{j}$ of each species is independent of time and position:

$$
F_{j}=-D_{j} \frac{d c}{d x}+\frac{D_{j}}{k T} z_{j} e^{\prime} c_{j} X=\text { constant },
$$

where $T$ denotes absolute temperature and $k$ Boltzmann's constant. We therefore obtain as many differential equations as there are ionic species. The field $X$ is at first unknown and must be determined from Poisson's equation,

$$
\frac{d X}{d x}=\frac{4 \pi e^{\prime}}{\varepsilon} \sum_{j} z_{j} c_{j}=-\frac{d^{2} \Phi}{d x^{2}},
$$

where $\Phi$ is the membrane potential. Normalizing, $\Phi(0)=0$, we obtain $\Phi(\delta)=\phi$ for the membrane potential difference. In the presence of an external e.m.f. a conduction current $I$ is maintained (taking inward current of positive ions to be positive)

$$
I=-e^{\prime} \sum_{j} z_{j} F_{j}
$$


$c_{j}(0+)$ and $c_{j}(\delta-)$ being prescribed through the partition coefficients $\Omega_{j}$ which depend on the membrane structure determining not only the phaseboundary potential differences but the ionic solubilities at the membrane boundaries as well:

$$
c_{j}(0+)=\Omega_{j} c_{j}(0-), c_{j}(\delta-)=\Omega_{j} c_{j}(\delta+) .
$$

For $N$ ionic species (2.4) and (2.5) supply the $2 N+1$ conditions needed to determine the $N$ fluxes and $N+1$ integration constants of the set of $N+1$ first order differential equations (2.2) and (2.3).

In considering the electrical characteristics of the squid giant axon it suffices to consider only monovalent ions $\left(\mathrm{K}^{+}, \mathrm{Na}^{+}, \mathrm{Cl}^{-}\right.$, and so on) as being possibly potential determining, $\left|z_{j}\right|=1$. We introduce the total concentration of all such ions, $C_{\text {tot }}=\sum_{j} c_{j}, C_{+}$, the total concentration of all positives, $C_{-}$, the total concentration of all negatives, $C_{-}=C_{\text {tot }}-C_{+}$and the dimensionless variables, all $O(1)$ in neuronal membranes,

$$
\psi=\frac{e^{\prime} \Phi(x)}{k T}, n=\frac{x}{\delta}, p=\frac{C_{+}}{C_{\text {tot }}}, n=\frac{C_{-}}{C_{\text {tot }}},
$$

and Poisson's equation becomes

$$
\begin{aligned}
\frac{-d^{2} \psi}{d n^{2}} & =\frac{\delta^{2}}{\lambda^{2}}(p-n), \\
\lambda^{2} & =\frac{\varepsilon k T}{4 \pi e^{\prime 2} C_{\text {tot }}},
\end{aligned}
$$

where $\lambda$ is the Debye length in the membrane.

We distinguish two limiting cases of (2.7).

(a) If $\delta^{2} \ll \lambda^{2}, \frac{d^{2} \psi}{d^{2}} \simeq 0$ even if $p \neq n$; the ions have low solubility in this the thin-membrane limit [3]. Though the calculated difference between the concentrations of positive and negative ions is finite, it is multiplied by the small parameter and the gradient of the 
field remains negligible. Poisson's equation is replaced by $X(x)=$ constant, that is, the Goldman [5] spatially-uniform, field limit. This case is considered by some authors, notably Katz [13], to be appropriate for neuronal membranes.

(b) If $\lambda^{2} \ll \delta^{2},(2.7)$ gives upon multiplication by $\lambda^{2} / \delta^{2}$, $p \simeq n$, even if $d^{2} \psi / d^{2} \neq 0$. Poisson's equation is now replaced by the condition of electrical neutrality, $\sum_{j} c_{j}(x)=0$. This corresponds to the thick-membrane, high solubility limit and is in effect Planck's argument [18] in modern form. Though the field calculated from the simplified set (2.2) and (2.3) is not uniform, the field gradient is multiplied by the small parameter $\lambda^{2} / \delta^{2}$, and the corresponding charge density remains negligible. Some authors (for exsmple, Tasaki [20]) consider this case to be the relevant one for some neuronal membranes.

If we suppose that the electrical characteristics of axonal membranes are deducible in terms of simple electrodiffusion of ions across a homogeneous membrane (which is effectively assumed in the discussion of limits (a) and (b) above) then we may make the following observations: the results of the above two limiting cases coincide if and only if $C_{\text {tot }}\left(0^{+}\right)=C_{\text {tot }}\left(0^{-}\right)$, in which case it is easy to show that Planck's field too is uniform. Making the assumption that the $\Omega_{j}$ are equal (see later) this is the situation in the natural axon (since the internal and external ionic strengths are equal to within a few percent, the concentration of polyvalent ions being slight) and it is only in perfusion experiments with changed ionic strength that the field structure is different in the two limits. In this case it is also easy to demonstrate that only Planck's limit can yield a non-uniform distribution of the field $X(x)$ which arises from the need for the individual fluxes to be independent of time and position in the steady state: where ionic strength is reducea, the electric field must be increased at the expense of the electric field elsewhere. Hence in the Planck limit, decreasing the axoplasmic ionic strength (by perfusion) increases the field near the inner surface of the membrane $(x=0+)$ which would render the high-field models of the excitable membrane still tenable. 
On these considerations, if limit (a) were the actual situation in the axonal membrane, a high resting field could exist only if the measured membrane potential difference were not the potential difference across the membrane. Thus, for example, if adsorbed negative charges on the inner surface of the membrane together with their counterions in the axoplasm are assumed to form an additional double layer partially masking the true membrane potential difference and increasing with decreasing axoplasmic ionic strength, then a large range of even uniform membrane fields may be postulated, depending on the magnitude chosen for the adsorbed negative charge.

However, the non-aqueous, non-ionizing medium of the axonal membrane interior [3] makes the unassisted passage of inorganic ions across the membrane extremely unlikely. We will show in the subsequent sections that the conclusions and implications of the previous two paragraphs must be strongly modified when facilitated passive ion transport occurs through well-defined pores exhibiting selectivity towards the current-carrying species. Subject to molecularly interpretable a priori assumptions about the mode of such transport of these ions through the channels, we will show that the various linear and nonlinear behaviour of the IC-V relationships exhibited by axonal membranes is deducible from our theory.

\section{A model of the linear squid giant axon}

We first propose a model of facilitated ion transport across the squid giant axonal membrane in a natural ionic environment and we show that this model reproduces the observed linearity of the IC-V [11, 12] of this axon, and leads in fact to the Hodgkin-Huxley formulation of the natural squid giant axon's electrical properties.

We suppose that $I_{j}$, the $j$ th component of the total axonal membrane conduction current $I$, is carried through the membrane via vell-defined pores which conduct the ionic species $j$ only (for the sake of simplicity we will understand, in considering the squid giant axon, that $j$ refers to either $\mathrm{K}^{+}$or $\mathrm{Na}^{+}$, though the theory may obviously be extended to include other conducting ions of either positive or negative charge). In the steady state, from (2.2), 


$$
-\frac{d c_{j}^{+}}{d x}+\frac{e^{\prime}}{k T} X_{j} c_{j}^{+}=-A_{j},
$$

where $A_{j}$ is independent of time and position, and $X_{j}(x)$ is the electric field in a $j$-pore. Furthermore, we suppose that the motion of these $j$-ions through the membrane is partially or totally compensated by a mobile, negatively charged, "carrier" ion of charge $\bar{z}_{j}^{-} e^{\prime}$ and concentration $\bar{c}_{j}(x)$ which is confined to the membrane and does not contribute to either $I_{j}$ or $I$ :

$$
-\frac{d c_{j}^{-}}{d x}+\frac{z_{j}^{-} e^{\prime}}{k T} X_{j} c_{j}^{-}=-B_{j},
$$

where $B_{j}$ is independent of time and position and

$$
c_{j}^{+}(x)=c_{j}^{-}(x)=c_{j}(x), \quad 0 \leq x \leq \delta .
$$

(3.1)-(3.3) give

$$
\begin{aligned}
\frac{d c_{j}}{d x} & =\frac{B_{j}^{-z_{j}^{-} A}}{1-z_{j}^{-}}, \\
X_{j} & =\frac{k T}{e^{\prime}} \frac{B_{j}^{-A} j}{\left(1-z_{j}^{-}\right) c_{j}},
\end{aligned}
$$

and

$$
c_{j}(x)=c_{0 j}+\frac{x}{\delta}\left(c_{\delta_{j}}-c_{0 j}\right),
$$

where

$$
c_{0 j} \triangleq c_{j}(0+), \quad c_{\delta j} \triangleq c_{j}\left(\delta_{-}\right) .
$$

It follows from (2.4) and (3.5) that

$$
X_{j}=\frac{k T}{e^{\prime} c_{j}}\left(\frac{-I j}{e^{\prime} D_{j}}+\frac{c_{\delta j}{ }^{-c} 0 j}{\delta}\right) \text {, }
$$

so that the membrane potential difference is

$$
\phi=\phi_{j}\left(1-\frac{I_{j} \delta}{e^{\prime} D_{j}\left(c_{\delta j}{ }^{-c_{0 j}}\right)}\right),
$$


whence

$$
\begin{aligned}
& I_{j}=g_{j}\left(\phi-\phi_{j}\right), \\
& g_{j}=e^{\prime} D_{j}\left(c_{0 j}-c_{\delta j}\right) / \delta \phi_{j}, \\
& \phi_{j}=\frac{k T}{e^{\prime}} \log \left(\frac{c_{0 j}}{c_{\delta j}}\right) .
\end{aligned}
$$

From (3.10)-(3.12) we note the following cases:

(i) when $c_{0 j}>c_{\delta j}, \phi_{j}>0$ (for example, for the $\mathrm{K}$ currentconducting system) and $I_{j}<0$ for $\phi<\phi_{j}$, that is, the current is outwardly directed as observed for $I_{\mathrm{K}}$;

(ii) when $c_{\delta j}>c_{0 j}, \phi_{j}<0$ (for example, for the Na currentconducting system), $I_{j}>0$ for $\phi>\phi_{j}$, and the current is inwardly directed as observed for $I_{\mathrm{Na}}$;

(iii) when either $c_{0 j}$ or $c_{\delta j} \rightarrow 0, g_{j} \rightarrow 0$ though $I_{j}$ is finite for finite $\phi$, approaching the values $c_{\delta j} e^{\prime} D_{j} / \delta$ and $-c_{0 j} e^{\prime} D_{j} / \delta$, respectively;

(iv) when $c_{0 j}=c_{\delta_{j}}, g_{j}=c_{0 j} e^{\prime 2} D_{j} / \delta k T(\neq 0)$, and $I_{j}=c_{0 j} e^{\prime 2} D_{j} \phi / \delta k T$, also non-zero for non-zero $\phi$.

The foregoing analysis is clearly not invalid in these important cases. In particular our definition of the chord conductance ((3.11)) holds in an external bathing solution which is free of the current-conducting species (for example, see Hodgkin and Huxley's remark [11, p. 480]).

In (3.10)-(3.12) we have recovered the famous Hodgkin-Huxley IC-V relationship for the natural squid giant axon and have given an interpretation of the chord conductances $g_{j}$ in terms of identifiable membrane parameters. In effect this has been achieved by applying three $a$ priori constraints :

I. complete specificity of the $j$-conducting pores towards 
ionic species $j$,

II. partial or total compensation of the charge of the transported ion for all $0 \leq x \leq \delta$,

III. such "electroneutrality" to be achieved with the aid of a mobile negative ion which makes no effective contribution to the measured conduction current $I_{j}$.

The question of how these conditions can be implemented in nerve basically involves the question of how simple inorganic ions can enter and traverse with relative ease the hostile, non-polar, low-dielectric constant, medium which constitutes the axolemma of a neuronal membrane. One model which satisfies the above three criteria depends on the existence of dipole chains traversing the membrane and parallel to the $x$ direction. A single approximately cylindrical pore could then be defined by a group of such chains. If the dipoles of the chains are normal to the direction of the chains with the negative poles all pointing in towards the centre of the pore, a core of polarizable negative charge is provided to compensate the positive charge of a $j$-ion moving through the $j$-pore. We suppose that on the approach of such an ion to the mouth of a pore, the negative core becomes polarized, providing a localized effective negative charge $z_{j}^{-}$ to offset the entrance of the ion to the pore, whereupon the ion is able to traverse the membrane accompanied by its negative "carrier" according to the equations of electrodiffusion as set out above. On the transference of the ion to the extramembrane medium the negative core relaxes to its equilibrium configuration, so that the net effect is the transfer of one positively charged ion across the membrane. If, in addition, the dipole core exhibits ion-selective properties (for example, by means of a specialized shape or size) the above three a priori criteria are all satisfied. For a successful transportation process the magnitude of $\boldsymbol{z}_{j}^{-}$ would of course depend upon the reduction needed in the (Born) solvation energy of the transported ion.

The well-known autonomy of the axonal membrane conductances in the natural squid giant axon is an obvious feature of the model both because of the physical separation of the pores and because of the proposed ionselective properties of the dipole carriers. The current-conducting ions 
do not co-determine the local electric field strength as in the case of the homogeneous membrane discussed in Section 2 , so that we now see the results of the previously-described perfusion experiments in a new light.

Our model predicts that changes in the ionic environment of the nerve membrane could affect its excitability through changing its $g^{\prime} s$ ((3.10)(3.12)). This arises directly through the factors depending on concentration or indirectly through the $D^{\prime} s$, which may themselves be functions of the local electric field strength redistributed by changes in the extramembrane medium. It is therefore possible that, for mechanisms for the control of ionic permeability in nerve which operate only in high fields, membrane excitability could be maintained in the face of a low internal potassium concentration (reduced $\phi$ ) provided $X_{\mathrm{Na}}(x)$ remains locally high despite a low average value. Thus the value of $\mathrm{X}_{\mathrm{Na}}(0+)$ (from (3.5), large for the natural squid giant axon since $c_{\mathrm{ONa}} \ll c_{\delta \mathrm{Na}}$ ) might, for example, be maintained near its natural value by increasing the concentration of external sodium as internal potassium is reduced. However, in the perfusion experiments actually performed (for example, [17]) where internal $\mathrm{K}^{+}$is reduced, excitability of the membrane should be lost, according to our model, for a sufficiently low concentration regardless of whether the experiment is performed at high or low internal ionic strength, because no favourable redistribution of $X_{\mathrm{Na}}(x)$ could occur in either case. In the context of this article, and on the assumption of a high-field mechanism for the control of membrane conductance, the observed continuance of excitability at low internal ionic strength would require a masking layer of the Hodgkin and Chandler type [10]. It follows that any test of our model of membrane conductance, such as the one outlined above should not be executed at low ionic strength.

An important implication of the foregoing model arises in connection with the construction of models of the permeability control process in nerve. On the ideas suggested above, the remarkable properties of the nerve impulse would arise solely from the dependence of the $D^{\prime} s$ on the time course of $\phi$. Presumably these diffusion coefficients have this functional dependence through some voltage-operated gating mechanism or mechanisms which control the passage of ions through the conducting pores; one possible gating mechanism has been suggested by the author $[14,15]$. 
In our model of facilitated ionic transport the dipole chains would of course operate independently of any such gating mechanism.

Finally we note that the assumption of a steady state in the flux of a single conducting pore $((3.1)$ and (3.2)) avoids undesirable transients in $I_{j}$; for example, if the closed gates of a pore were simply a localized obstruction to conducting ions at some $x=a$ then in the steady state there would be a Maxwell-Boltzmann build up of conducting ions in a closed pore for $x>a$ and a Maxwell-Boltzmann depletion for $x<a$ (assuming the membrane field is the resting field). If the gates are now suddenly opened (for examples, as a result of a rapid depolarization of the axon) these ionic distributions would give rise to a complicated redistribution of the conducting ions in the pore with obvious undesirable effects on $I_{j}$. If however the gates are such as to prevent free movement of conducting ions along the entire pore when the gate is closed, the concentration profile of the ions across the membrane would be "frozen" in position when a gate is suddenly closed. The steady state situation described by (3.1) and (3.2) then holds for both states of the gating mechanism. This suggests that the gate has the form of a spiral or helix stretching the entire length of the pore and completely enclosing it. A transition of the helix to a larger pitch might render the pore closed by restricting the passage of ions through the now narrower pore.

\section{The Goldman relation for axonal current}

Unlike the squid giant axon, other axonal membranes, for example, that of the node of Ranvier in frog nerve, exhibit a nonlinear IC-V even in a natural ionic environment [9]. We now show how our model of facilitated ion transport can result in such a nonlinearity.

We suppose here that "electroneutrality" again constrains the passage of current-carrying ions through the membrane, but in a form different to that of the preceding section: we replace (3.3) by the new condition

$$
\text { (4.1) } \quad \sum_{j} c_{j}^{+}(x)=\sum_{j} c_{j}^{-}(x)=S(x), 0 \leq x \leq \delta,
$$

where the first summation is over all the positive ions contributing to the total conduction current, and the second summation is over their 
corresponding negative carriers of effective valence $\overline{z_{j}}$ whose properties were discussed in section 3. We now have in the steady state (3.1) and (3.2) replaced by

$$
\begin{gathered}
-\frac{d c_{j}^{+}}{d x}+\frac{e^{\prime}}{k T} X c_{j}^{+}=-A_{j}^{\prime}, \\
-\frac{d c_{j}^{-}}{d x}+\frac{z_{j}^{-} e^{\prime}}{k T} X c_{j}^{-}=-B_{j}^{\prime},
\end{gathered}
$$

where $X(x)$ is the electric field corresponding to condition (4.1) and $A_{j}^{\prime}$ and $B_{j}^{\prime}$ are constants. From $(4.1)-(4.3)$,

$$
\begin{array}{r}
-\frac{d S}{d x}+\frac{e^{\prime}}{k T} X S=-\sum_{j} A_{j}^{\prime}=-A, \\
-\frac{d S}{d x}+\frac{z^{-} e^{\prime}}{k T} X S=-\sum_{j} B_{j}^{\prime}=-B,
\end{array}
$$

if

$$
z_{\mathrm{K}}^{-}=z_{\mathrm{Na}}^{-}=\ldots=z^{-}
$$

Hence

$$
\begin{aligned}
\frac{d S}{d x} & =\frac{B-z^{-} A}{1-z^{-}}, \\
S(x) & =S_{0}+\frac{x}{\delta}\left(S_{\delta}-S_{0}\right),
\end{aligned}
$$

where

$$
S_{0} \triangleq S(0+), S_{\delta} \triangleq S\left(\delta_{-}\right)
$$

It follows that

$$
X=\frac{k T}{e^{\prime}} \frac{B-A}{\left(1-z^{-}\right) S},
$$

and so the membrane potential difference is, from (4.7)-(4.10),

$$
\phi=\frac{k T^{\prime} \delta}{e^{\prime}\left(1-z^{-}\right)} \frac{A-B}{S_{\delta}-S_{0}} \log \frac{S_{\delta}}{S_{0}} .
$$

Now if we suppose, as before, that only positive ions contribute to 
the total axonal conduction current, (4.4)-(4.11) yield

$$
\frac{d U}{d x}+\frac{A-B}{\left(1-z^{-}\right)} \frac{U}{S}=\frac{I}{e^{\prime}},
$$

where

$$
U=\sum_{j} D_{j}^{+} c_{j}^{+}
$$

$$
I=e^{\prime} \sum_{j} D_{j}^{+} A_{j}^{\prime}
$$

Setting

$$
\xi=\left(S_{\delta} / S_{0}\right)^{\delta(A-B)\left\{\left(1-z^{-}\right)\left(S_{\delta}-S_{0}\right)\right\}},
$$

we obtain on integrating (4.12),

$$
\xi=\frac{I \delta S_{0}-e^{\prime} U_{0}(1+\nu)\left(S_{\delta}-S_{0}\right)}{I \delta S_{\delta}-e^{\prime} U_{\delta}(1+\nu)\left(S_{\delta}-S_{0}\right)},
$$

$$
v=\frac{\delta(A-B)}{\left(1-z^{-}\right)\left(S_{\delta^{-}} S_{0}\right)} \text {. }
$$

In the natural squid giant axon $\sum_{j} c_{j}^{+}(0-) \simeq \sum_{j} c_{j}^{+}(\delta+),[9]$, so that if we set $\Omega_{\mathrm{K}}=\Omega_{\mathrm{Na}}$ it follows that $S_{0} \simeq S_{\delta}$ and so from (4.11) and (4.16),

$$
I \simeq \frac{e^{\prime 2} \phi}{k T \delta} \frac{U_{\delta} e^{e^{\prime} \phi / k T}-U_{0}}{e^{e^{\prime} \phi / k T}-1}
$$

In fact this relation holds to very good accuracy for deviations of $\Omega_{\mathrm{K}} / \Omega_{\mathrm{Na}}$ from unity by as much as $20 \%$. Also since the density of sodium pores is much greater than that of $K$ pores [8] we have as a consequence of this that $\Omega_{P K} / \Omega_{P N a} \gg 1$ where the $\Omega_{j}$ is related to the partition coefficient $\Omega_{P j}$ in individual $j$-pores and their areal density $\rho_{j}$ by

$$
\Omega_{j}=\Omega_{P j} \rho_{j}
$$

In (3.10)-(3.12) and $(4.18)$ we have two distinct relationships for membrane current as a function of potential difference and we next seek an 
interpretation of this difference in terms of the two limits discussed in Section 2 and the two "electroneutrality" conditions (3.3) and (4.1).

Now for the squid giant axon the estimate of the distance between sodium conducting pores is about $450 \AA$ and that between potassium conducting pores is about $1400 \AA$ [8], so that we may take the mean distance $d$ between current conducting pores (of both types) to be such that $d \gg \delta \simeq 70 \AA$. If the Planck domain of validity applied in the neuronal membrane under consideration, $\lambda$ would certainly be much less than $d$. The passage of $K$ ions through the membrane is then quite independent of the passage of $\mathrm{Na}$ ions through $i t$, since no interaction is then possible between the two currents. The measured $j$ component of $I$ from a test patch of membrane (of dimension $\gg d$ ) is then simply the sum of the contributions from the individual (identical) $j$-pores in this patch; the relevant "electroneutrality" condition is evidently that given by (3.3) and the relationship between current and voltage is that described by (3.10)-(3.12). This is clearly the case in which the conduction currents are observable microscopicalzy (that is, over patches the size of a cross section of a conducting pore).

However if the Goldman domain applies, $\lambda$ could be greater than $d$, and interaction between currents in adjacent pores would then occur. In this case (3.3) is clearly inappropriate because the current from the $j$-pores is not observable microscopically as before; because of interference from neighbouring pores, the measurement of membrane current is now possible only at the macroscopic level (that is, over areas the size of the entire membrane test patch) where the relevant "electroneutrality" condition is evidently that given by (4.1), which therefore now replaces (3.3) in our a priori criteria whenever $\lambda>d$. Thus the $x_{j}$ of (3.5) is written with the subscript $j$ in order to emphasize its microscopic character and the $X$ of (4.10) has no such subscript in view of its essentially macroscopic nature.

Notwithstanding this amendment of our a priori constraints, however, we still assume complete specificity of $j$-channels towards the $j$ th ionic species as before. Thus $D_{j} c_{j}$ is taken to be the only non-vanishing current term in $j$-channels, and so, from (4.18), 


$$
I_{j} \simeq D_{j} c_{0 j} \frac{e^{\prime 2} \phi}{k T \delta} \frac{\left(c_{\delta j} / c_{0, j}\right) e^{e^{\prime} \phi / k T}-1}{e^{e^{\prime} \phi / k T}-1},
$$

the so-called Goldman expression which has been found to describe the data for the IC-V relationship for the frog node of Ranvier [9].

We therefore suggest that the linearity property exhibited by the natural squid giant axon is a consequence of the independence of the $I_{j}$, that is, of the non-interaction between current-carrying channels. Though the Planck limit may still apply in this case, it seems unnecessarily severe in the light of the above argument, which merely requires that the linearity property of a neuronal membrane applies for $\lambda<d$. The nonlinearity as exhibited by the IC-V relationship of, for example, frog node of Ranvier, we interpret as resulting from the case $\lambda>d$, which could arise by a decrease of ionic solubility (increase in $\lambda$ ) or by a decrease of $d$, as compared to the case of the natural squid giant axon. At a node of Ranvier the peak inward current density during excitation is some 10 times that of the squid giant axon [9]. Such an increase could be achieved most simply by a greater density of current-carrying pores giving rise to a smaller value of $d$ and a possible transition to the case $\lambda>d$. In view of the estimated value of $d$ above, this strongly suggests that the Planck limit may be inappropriate even for the natural squid giant axon. Our model of ionic transport through neuronal membranes requires that the electric field should be a function of position [(3.5) and (4.10)] so that the model bears superficial resemblance to both the Planck and Goldman models.

\section{Mappings of the axonal electric fields}

In order to compute the axonal elelctric field predicted by (3.1)(3.12) we have first to solve Laplace's equation for the potential $\Phi$ given as boundary conditions the potential $\Phi_{P j}$ along the axis of a $j-$ pore and the potential difference $\phi$ across the membrane where, from (3.8) and $(3.12)$,

$$
\Phi_{P j}=\frac{\phi}{\log \left(c_{\delta} / c_{0}\right)_{j}} \log \left\{1+\frac{x}{\delta}\left[\left(c_{\delta} / c_{0}\right)_{j}-1\right]\right\} .
$$


We approximate the membrane to a parallel-plate condenser with potential difference $\phi$ across the plates and take cylindrical coordinates $r, \theta, x$ with origin in the inner plate and the $x$ axis normal to the plates (as in the previous sections). Since the diameter of a conducting pore must be small ( $\simeq 5 \AA)$ compared to $\delta$, we shall approximate a pore by the straight-line segment $0 \leq x \leq \delta, r=0$ and set $\phi=1$. Thus once $\Phi(r, \theta, x)$ has been computed we can obtain the solution for any potential difference across the membrane by multiplying $\Phi$ by the appropriate factor. To make the problem determinate we assume that the potential is linear (corresponding to a uniform electric field) across the membrane, far from the conducting pore.

Since $\Phi$ has azimuthal. symmetry, Laplace's equation reduces to

$$
r \frac{\partial^{2} \Phi}{\partial r^{2}}+\frac{\partial \Phi}{\partial r}+r \frac{\partial^{2} \Phi}{\partial x^{2}}=0
$$

We construct a grid in the $r-x$ plane with step length of $h$ in the $r$ direction and $k$ in the $x$ direction. The typical mesh-point is denoted by $\Phi_{m, n}$ where $m=r / h, n=x / k$ and using the central difference formula for numerical derivatives (5.2) becomes

$$
b \Phi_{m-1, n}+c \Phi_{m, n-1}+a \Phi_{m, n}+c \Phi_{m, n+1}-\Phi_{m+1, n}=0
$$

where

$$
\left\{\begin{array}{l}
a=2 r / d\left(1 / h^{2}+1 / k^{2}\right), \\
b=\left(1 / 2 h-r / h^{2}\right) d, \\
c=-r /\left(k^{2} d\right) \\
d=(0.5+r / h) / h .
\end{array}\right.
$$

If $m$ and $n$ are allowed to vary appropriately (5.3) and (5.4) become a set of linear equations in the unknowns $\Phi_{m, n}$ which is solved using the Gauss-Seidel. iterative scheme with relaxation. Once a sufficiently accurate numerical approximation to $\Phi$ has been found, the electric field

$$
X=-\nabla \Phi
$$

must be extracted from the discrete set of all the $\Phi_{m, n}$. The central difference method, while being the simplest, is the least reliable 


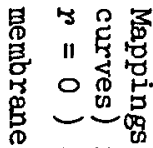

숭ㅇㅇㅇㅇㅇ

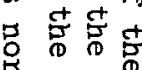

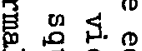

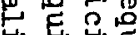

os 2 .

$\stackrel{0 .}{\circ}$ \&

웅

$\pm \mathscr{0}$ 즌

索占 ! 员

政要

ॠ范

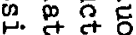

吕 E 占

pio

它

$+85$

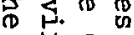

两

雨号号

它要

点索

5

可品

\& 0 的

"它索㕝

0 象

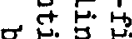

员.

5. in

$\infty$ 边.

十紧些.

要边

象过品

S

点 0 咅

IN

రृ

फ.

䒠皆的点

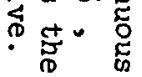

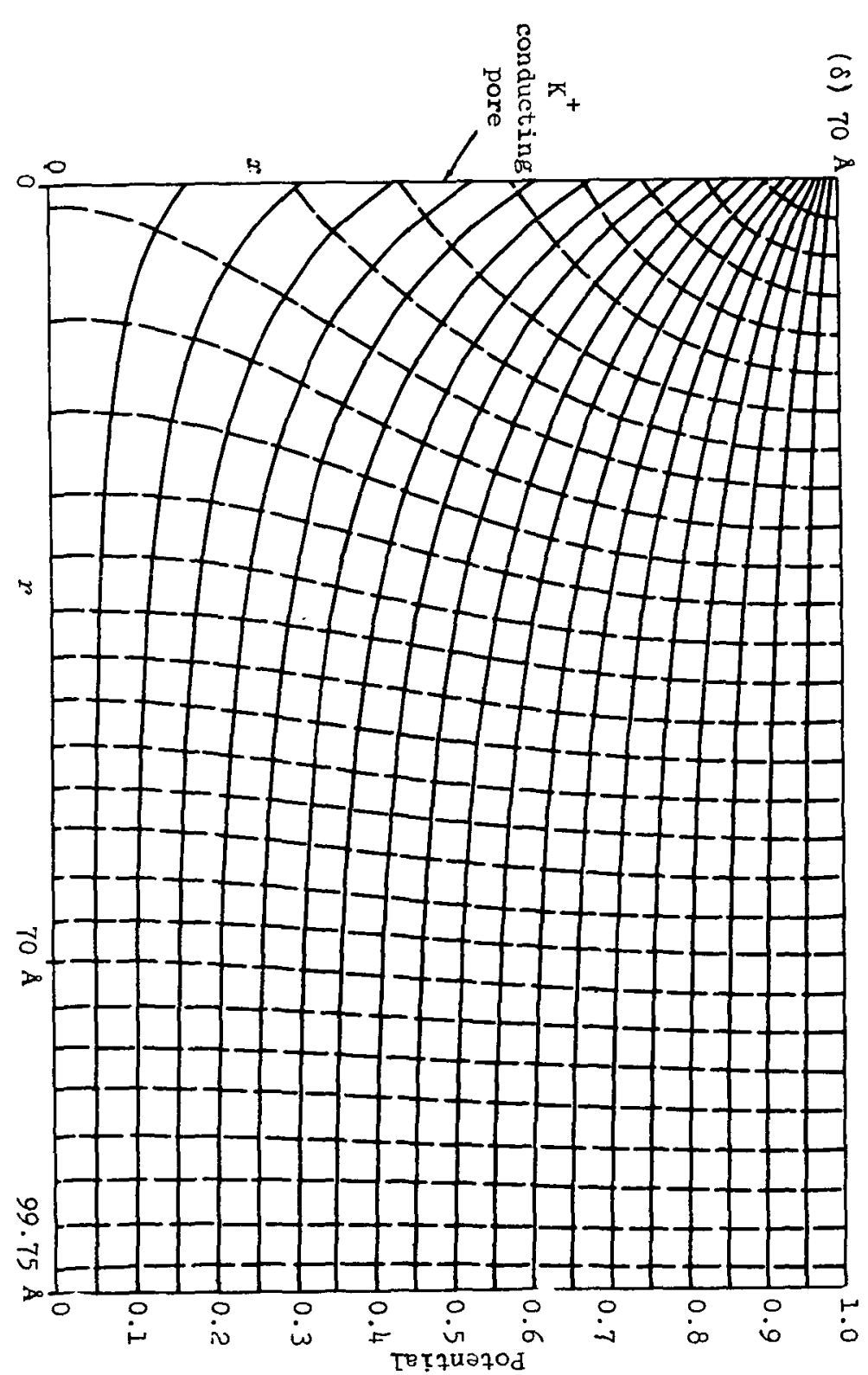




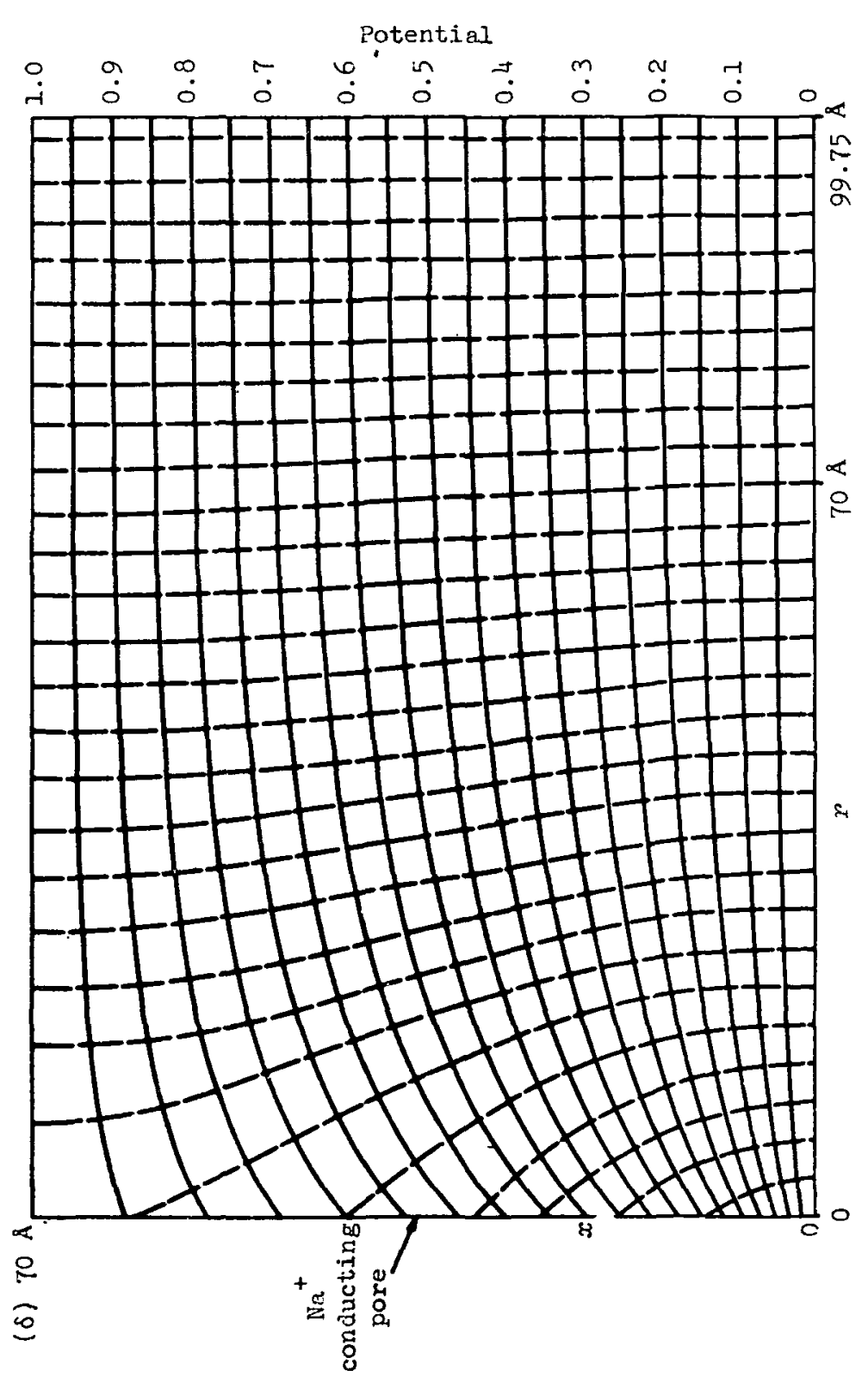

鸹

孚 $v 1 \stackrel{0}{0}$

至 \&

un VI ब

.

$-+\stackrel{2}{0}$

凹

西安

त ब

त्

$\rightarrow \cdot r$.

I

$0 \notin \infty$

然宁

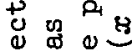

बठ

总

( )

कू

员牙

०ै 010

:

等苛年

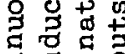

갱 0

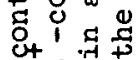

- 0 I

के क वै

芯出呆。

मै

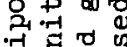

굴

क्त व

过

†密

4. द्य क्त

䑻

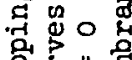

题

i

璐 
approach, so a smooth function is fitted to the points $\Phi_{m, n}$ and differentiated to find the field. The most reliable curve-fitting procedure for the latter is found to be the cubic spline; this is fitted independently in the $r$ and $x$ directions, so that the derivatives of the fitted functions yield the components $-x_{p}$ and $-X_{x}$ respectively. The difficulty in deciding on the best fit is that the exact solution for $X$ is not of course known. However, a useful check may be made of the fit in the $x$ direction since the field is specified exactly all along the pore. The fit is applied to values of $\Phi_{P}$ at intervals of $2.5 \AA$ and the resulting field may be compared with the exact value. For the potassium system, the worst error is $14.5 \%$ at $x=70 \AA$, but at $x=65 \AA$ this has reduced to $2.8 \%$. For the sodium system, the worst error is $1.2 \%$ at $x=0$ which reduced to $0.1 \%$ at $5 \AA$. These errors can be reduced if necessary, the only criterion being the cost of computer time.

The results of the computations are shown in Figures 1 and 2 . These show that, in the vicinity of the conducting pores of the natural squid giant axon, $\left(\left(c_{0} / c_{\delta}\right)_{\mathrm{K}}=40,\left(c_{0} / c_{\delta}\right)_{\mathrm{Na}}=1 / 9.2\right)$ the electric field is far from uniform, for example, near the inner edge of the membrane in the vicinity of a sodium conducting pore the electric field has a much higher intensity than elsewhere. In the experiment suggested in Section 3 (that is, the possible maintenance of a high value of $X_{\mathrm{Na}}(0+)$ by simultaneously increasing the concentration of external $\mathrm{Na}^{+}$and decreasing the internal concentration of $\mathrm{K}^{+}$) the field in the vicinity of the sodium pores becomes more deformed as the field in the vicinity of the potassium pores tends to uniformity. We suggest that these two effects may offset each other in their effect on the excitability of the axon.

The author has suggested [16] that these nonuniformities in the axolemmic electric fields may have important functional significance in the propagation of the nervous impulse.

\section{A model of the nonlinear squid giant axon}

The preceding analysis assumes that a $j$-channel conducts only $j$-ions. If however, mobile species other than $j$-ions are present in $j$-channels this analysis must be modified. We consider an example of this 
in relation to the choline-seawater experiments of Hodgkin and Huxley [11].

Choline has been employed in the separation of the axonal membrane conduction current into its potassium and sodium components on the assumption that, when it replaces sodium in the bathing solution, the resting potential is little affected and choline makes no contribution to $I_{\mathrm{K}}$ [11]. The Hodgkin-Huxley choline-seawater experiments show that the squid giant axon exhibits the striking property change from linearity in sodium (natural) seawater to nonlinearity in sodium-deficient bathing solutions.

In terms of our model of the membrane conduction process, this result could be interpreted in two ways: either

(1) the substitution of choline for sodium in the extracellular medium is accompanied by a transition to the case $\lambda>d$, and our treatment of Section 4 applies, or

(2) though $\lambda<d,(3.3)$ is replaced by (4.1) in individual pores because of the presence in the pores of more than one mobile ionic species, namely, $\mathrm{Na}$ ions and choline ions in what is solely a sodium pore in the natural axon.

(For example, Hille [6, 1] demonstrated that mobile ions other than sodium can penetrate channels when the axon is placed in an unnatural ionic environment.) In other words, at the expense of a relaxation of a priori constraint $I,(4.1)$ and the theory of Section 4 have a microscopic interpretation in this experiment.

In case (1), $\lambda$ could presumably be increased by the removal of most of one of the conducting species, that is, sodium from the membrane. However, this interpretation would mean that the value of the membrane $\lambda$ is determined largely by the concentration of the conducting ions. This seems unlikely in view of the sparcity of conducting pores.

The alternative, case 2, appears to be more likely because of the large concentrations of extracellular choline $(\simeq 460 \mathrm{mM})$ used in these experiments. Linearity of the IC- $V$ for potassium is preserved in choline solutions [11], so we simply assume that choline is excluded from $K$-pores but not from Na-pores; this ensures that neither the resting potential nor the potassium current is much affected by the replacement process (as 
observed).

If both choline and sodium ions are present in sodium pores we have from (4.15) and (4.16) the conduction current flowing in the Na-pores, $I_{\mathrm{Na}}$, given by

(6.1) $\quad I_{\mathrm{Na}}=\frac{e^{\prime}}{\delta}\left(\left(S_{\delta} / S_{0}\right)-1\right)\left(\frac{\phi e^{\prime}}{k T 10 g\left(S_{\delta} / S_{0}\right)}+1\right) \frac{U_{\delta} e^{e^{\prime} \phi / k T}-U_{0}}{\left(S_{\delta} / S_{0}\right) e^{e^{\prime} \phi / k T}-1}$,

where in this case

$$
U_{\delta}=D_{\mathrm{Na}} c_{\delta \mathrm{Na}}+D_{\mathrm{Ch}} c_{\delta \mathrm{Ch}}
$$

$$
U_{0}=D_{\mathrm{Na}} c_{\mathrm{ONa}} \text {, }
$$

$$
s_{\delta}=c_{\delta \mathrm{Na}}+c_{\delta \mathrm{Ch}}
$$

$$
S_{\mathrm{O}}=c_{\mathrm{ONa}}
$$

In Figure 3 we show the theoretical "sodium" curves versus depolarization from the natural resting potential, drawn for different values of the extracellular sodium concentration. Exact comparison with experiment is precluded by the uncertainty in the experimental values of $c_{\mathrm{Na}}(\delta+)$ (since measurements, beginning at data point 1 , were started before all the sodium had diffused away from the nerve [11]) but the theory is clearly in good agreement with experiment. In the curve fitting of Figure 3, $D_{\mathrm{Na}} / D_{\mathrm{Ch}}=100$ and for choline seawater $c_{\delta \mathrm{Ch}} / c_{\mathrm{ONa}} \simeq 6.64$. Values of $D_{\mathrm{Na}} / D_{\mathrm{Ch}}$ much different to this would lead to either unacceptably large values of $D_{\mathrm{Na}} c_{\mathrm{ONa}}$ (see later) or $c_{\mathrm{OCh}} / c_{\mathrm{ONa}}$. The values actually chosen for $D_{\mathrm{Na}} / D_{\mathrm{Ch}}$ and $c_{\delta \mathrm{Ch}} / c_{\mathrm{ONa}}$ would mean that though the affinity of the sodium "carriers" for choline is rather high (comparable to that for sodium itself, since in normal seawater $c_{\delta \mathrm{Na}} / c_{\mathrm{ONa}}=9.2$ ) the mobility of choline in a sodium pore is negligibly small in comparison with that of $\mathrm{Na}^{+}$. This would mean in turn that choline does not contribute directly to $I_{\mathrm{Na}}$ but affects $g_{\mathrm{Na}}$ through distortion of $X_{\mathrm{Na}}(x)$.

Furthermore we are able to determine the important membrane 


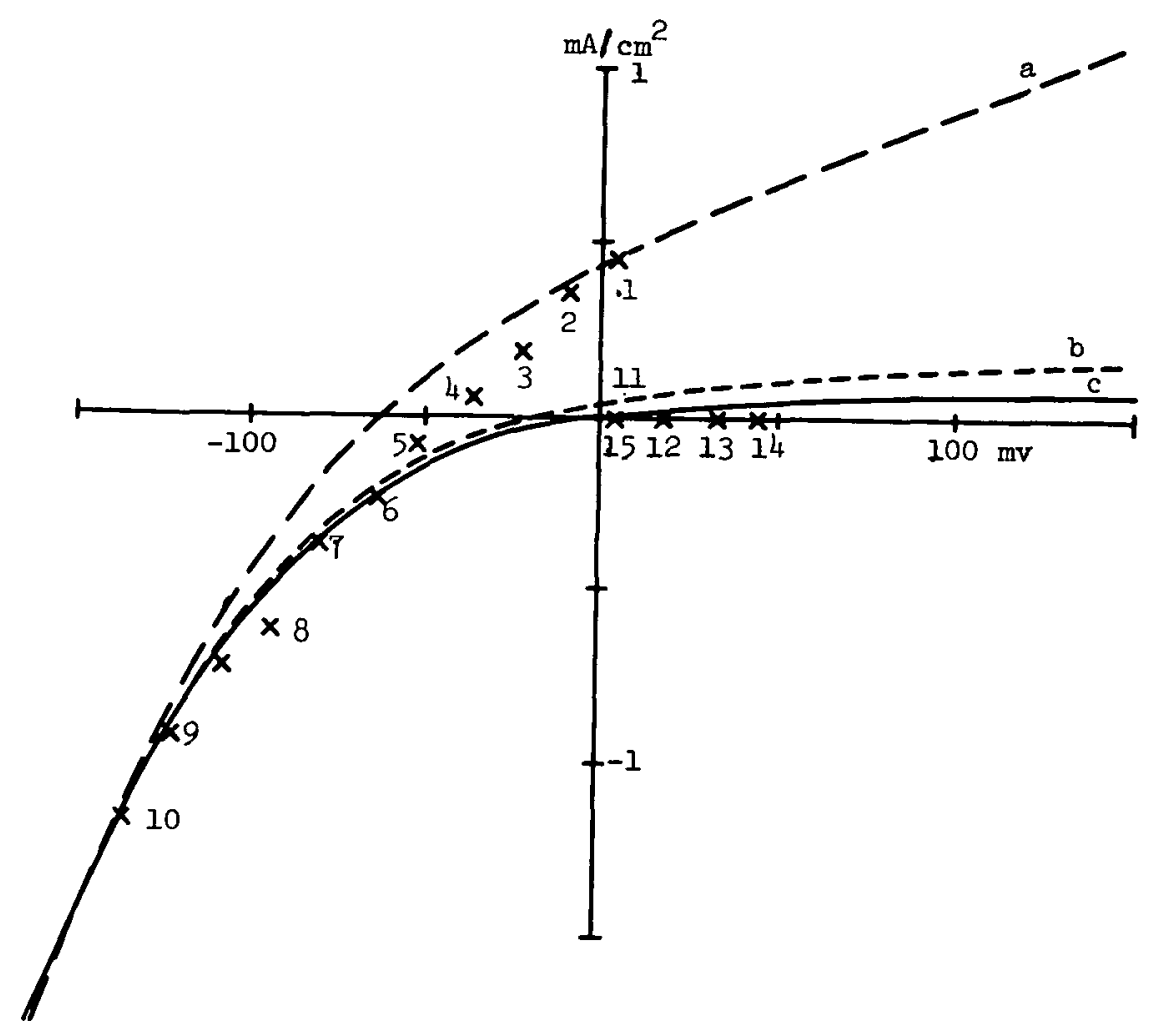

FIGURE 3. Sodium instantaneous current-voltage relationship in sodiumcholine seawater bathing solutions. The curves are plotted from equation (6.1); for curve (a) the concentration of external sodium is $10 \%$ of that of normal seawater, for curve (b) it is $1 \%$, and for curve (c) choline seawater is the external medium.

The abscissa represents voltage displacement from the resting potential; negative voltages represent depolarization and positive voltages represent hyperpolarization. The crosses are experimental results for the squid giant axon in choline seawater, with the numbers indicating the order in which the measurements were taken (Figure 7 of [11]).

parameters $D_{j} c_{0 j}$ from our model: expressing the $c_{0}$ 's in moles per $\mathrm{cm}^{3}$, (i) from (3.11) and (3.12) and Figure 7 of [11],

$$
D_{\mathrm{Na}} c_{\mathrm{ONa}}=1.47 \times 10^{-15} \mathrm{~cm}^{-1} \mathrm{sec}^{-1} \text {, }
$$


( $5^{\circ} \mathrm{C}$, initial depolarization $110 \mathrm{mV}$, in natural seawater), (ii) from Figure 3 above

$$
D_{\mathrm{Na}} c_{\mathrm{ONa}}=1.73 \times 10^{-15} \mathrm{~cm}^{-1} \mathrm{sec}^{-1}
$$

( $5^{\circ} \mathrm{C}$, initial depolarization $110 \mathrm{mV}$, in choline seawater), (iii) from (3.11) and (3.12) and Figure 12 of [11],

$$
D_{\mathrm{K}} c_{\mathrm{OK}}=13.4 \times 10^{-15} \mathrm{~cm}^{-1} \mathrm{sec}^{-1} \text {, }
$$

( $20^{\circ} \mathrm{C}$, initial depolarization $84 \mathrm{mV}$ in choline seawater). Bearing in mind that with equal partition coefficients $\left(\Omega_{\mathrm{K}}=\Omega_{\mathrm{Na}}\right) c_{\mathrm{OK}} / c_{\mathrm{ONa}}=8$ and that under the experimental conditions the sodium and potassium conducting systems are in similar states of excitation, results (i), (ii) and (iii) above are at least plausible. Exact comparison would depend on the construction of a detailed model of the ionic permeability-controlling systems. In addition, if we suppose that in the experiment associated with result (iii), $g_{\mathrm{K}}$ is increased by a factor $\simeq 75$ relative to its resting value [12] and if we take Cole's estimate [2] of the resting value of $D_{K}$ $\left(2.5 \times 10^{-10} \mathrm{~cm}^{2} \mathrm{sec}^{-1}\right)$, then (iii) yields $c_{\mathrm{OK}} \simeq 0.72 \mathrm{mM} /$ litre and an average value of conducting ion in the natural squid giant axonal membrane of $\simeq 0.4 \mathrm{mM} /$ litre and $\Omega_{\mathrm{K}}\left(=\Omega_{\mathrm{Na}}\right) \simeq 1.8 \times 10^{-3}$ at $20^{\circ} \mathrm{C}$.

\section{Ionic selectivity}

If sodium ions are present as impurities in the potassium-conducting system of the natural squid giant axonal membrane and vice versa, (4.1) representing our macroscopic "electroneutrality" condition can be given a microscopic interpretation much in the manner of the previous section, that is, it can be applied to individual pores even though we assume that no interaction between adjacent current-conducting pores occurs. Thus applying the analysis of Section 4 we have a result similar to $(6.1)-(6.5)$ :

(7.1) $I_{j}=\frac{e^{\prime}}{\delta}\left(\left(S_{\delta} / S_{0}\right)_{j}^{-1}\right)\left(\frac{\phi e^{\prime}}{k T \log \left(S_{\delta} / S_{0}\right)_{j}}+1\right) \frac{U_{\delta j} e^{e^{\prime} \phi / k T}-U_{0 j}}{\left(S_{\delta} / S_{0}\right)_{j} e^{e^{\prime} \phi / k T}-1}$, where 


$$
\begin{aligned}
& U_{\delta j}=\left(D_{\mathrm{Na}} c_{\delta \mathrm{Na}}+D_{\mathrm{K}} c_{\delta \mathrm{K}}\right)_{j}, \\
& U_{0 j}=\left(D_{\mathrm{Na}} c_{\mathrm{ONa}}+D_{\mathrm{K}} c_{\mathrm{OK}}\right)_{j}, \\
& s_{\delta j}=\left(c_{\delta \mathrm{Na}}+c_{\delta \mathrm{K}}\right)_{j}, \\
& S_{0 j}=\left(c_{\mathrm{ONa}}+c_{\mathrm{OK}}\right)_{j},
\end{aligned}
$$

with $j=K, \mathrm{Na}$.

Now, in the presence of impurity $(2)$ ions in a $j$-pore (that is, of $\mathrm{K}$ ions in a $\mathrm{Na}$ pore or vice versa) the known linearity of the IC- $\mathrm{V}$ relationship of the natural squid giant axon can be retained in (7.1) if and only if $D_{l} \simeq D_{j}$. (It turns out that the linearity of (7.1) is preserved for quite large deviations $(\simeq 20 \%)$ of $D_{l} / D_{j}$ from unity.? This approximation of $D_{l}$ to $D_{j}$ in a given pore could arise because, according to our model a $\mathrm{K}^{+}$or an $\mathrm{Na}^{+}$moves through the axonal membrane with the aid of an oscillating system of dipoles. The inertia of this system is clearly very much greater than that of the transported ion, so if the ion can move freely in the pore its mass or size will have little effect on the oscillation of the dipole system. As a result the mobility of a small monovalent ion in a pore will be essentially independent of its species. Such might not be true of a larger ion where friction at the "walls" of the pore might impede its motion through the membrane. Our analysis of section 6 indicates such a situation for choline in sodium pores.

The potential-determining character of $j$-ions in $j$-pores must therefore arise through the $j$-ions being the major concentration in these pores, that is, through $\Omega_{P l} \ll \Omega_{P j}$. Alternatively $\pi_{j} \gg 1$ where

$$
\pi_{j}=\left(\frac{\Omega_{P j}}{\Omega_{P l}}\right)_{j}
$$

From (7.1)-(7.5) the equilibrium potential for the system conducting ionic species $j$ is then

$$
\phi_{j}=\frac{k T}{e^{\prime}} \log \left(\frac{S_{0}}{S_{\delta}}\right)_{j}=\frac{k T}{e^{\prime}} \log \frac{\pi_{j} c_{j}(0-)+c_{\eta}(0-)}{\pi_{j} c_{j}(\delta+)+c_{\eta}(\delta+)},
$$


where if $j=K, \mathrm{Na}, \quad=\mathrm{Na}, \mathrm{K}$, respectively.

Since $c_{K}(\delta+) \ll c_{\mathrm{Na}}(\delta+)$ in the natural squid giant axon [9] it follows that

$$
\phi_{\mathrm{Na}} \simeq \frac{k T}{e^{i}} \log \frac{\pi_{\mathrm{Na}} c_{\mathrm{Na}}(0-)+c_{\mathrm{K}}(0-)}{\pi_{\mathrm{Na}} c_{\mathrm{Na}}(\delta+)}
$$

which is identical to the expression used by Chandler and Meves [4] and others if we identify $\pi_{\mathrm{Na}}$ with what some have chosen to call the ratio of the permeabilities of $\mathrm{Na}^{+}$and $\mathrm{K}^{+}$(in sodium pores) but which we will refer to as the selectivity coefficient of the sodium conducting system; similarly $\pi_{K}$ will be the selectivity coefficient of the potassium conducting system.

Taking $T=8^{\circ} \mathrm{C}$ and a natural ionic environment for the squid giant axon (7.7) gives $\phi_{\mathrm{Na}} \simeq-50 \mathrm{mV}$ if $\pi_{\mathrm{Na}} \simeq 48.6$ and $\phi_{\mathrm{Na}} \simeq-45 \mathrm{mV}$ if $\pi_{\mathrm{Na}} \simeq 18.4$. These values clearly provide an estimate of the bounds of $\pi_{\mathrm{Na}}$ which however will certainly vary with $T$ and species of squid (see also Brinley's comment [1]). A similar calculation for the potassium system at $8^{\circ} \mathrm{C}$ yields $\phi_{\mathrm{K}} \simeq 72 \mathrm{mV}$ if $\pi_{\mathrm{K}} \simeq 43.9$ and $\phi_{\mathrm{K}} \simeq 77 \mathrm{mV}$ if $\pi_{K} \simeq 69.2$. (We have assumed that each conducting species has equal activity coefficients in the external bathing solution and in the axoplasm; this is unlikely to be true [9] so these calculations need some slight. modification.)

It is important to observe that with $D_{l} \simeq D_{j},(7.1)-(7.5)$ reduce to (3.10)-(3.12) with $S_{\delta j}, S_{0 j}$ replacing $c_{\delta j}, c_{0 j}$ respectively. Thus employing (7.1) to describe the electrical characteristics of the natural squid giant axon instead of (3.10)-(3.12) is equivalent to using these latter equations but with the ratio $c_{\delta \mathrm{Na}} / c_{\mathrm{ONa}}$ reduced by $14 \%$ for $\pi_{\mathrm{Na}}=48.6$ and by $30 \%$ for $\pi_{\mathrm{Na}}=18.4$ and with the concentration ratio $c_{\delta \mathrm{K}} / c_{\mathrm{OK}}$ increased by $104 \%$ for $\pi_{\mathrm{K}}=43.9$ and $66 \%$ for $\pi_{\mathrm{K}}=69.2$. Hence (3.10)-(3.12) are oversimplified but nevertheless represent reasonable expressions to employ in order of magnitude calculations; however, in theories concerned with the microscopic properties of ionic 


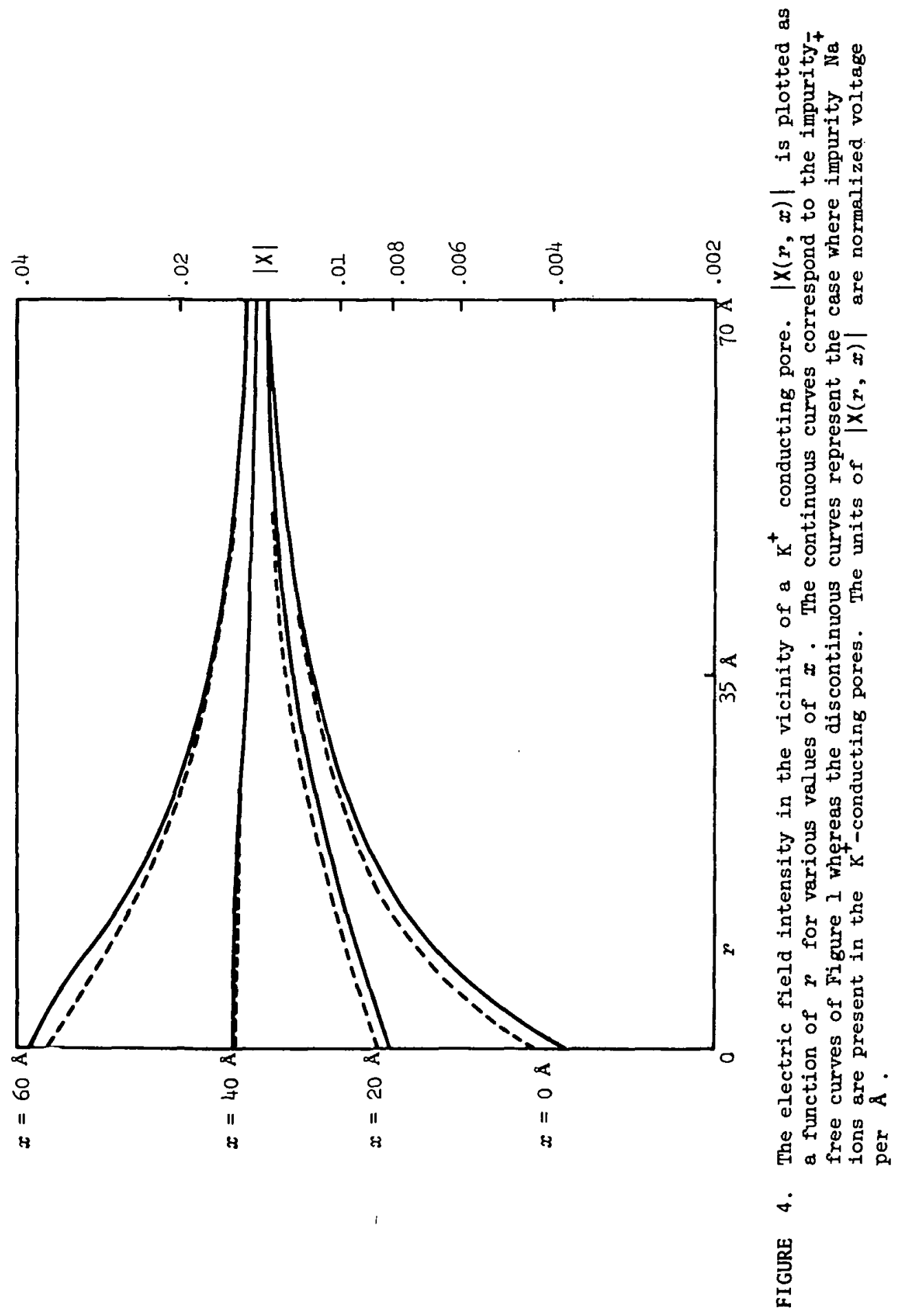




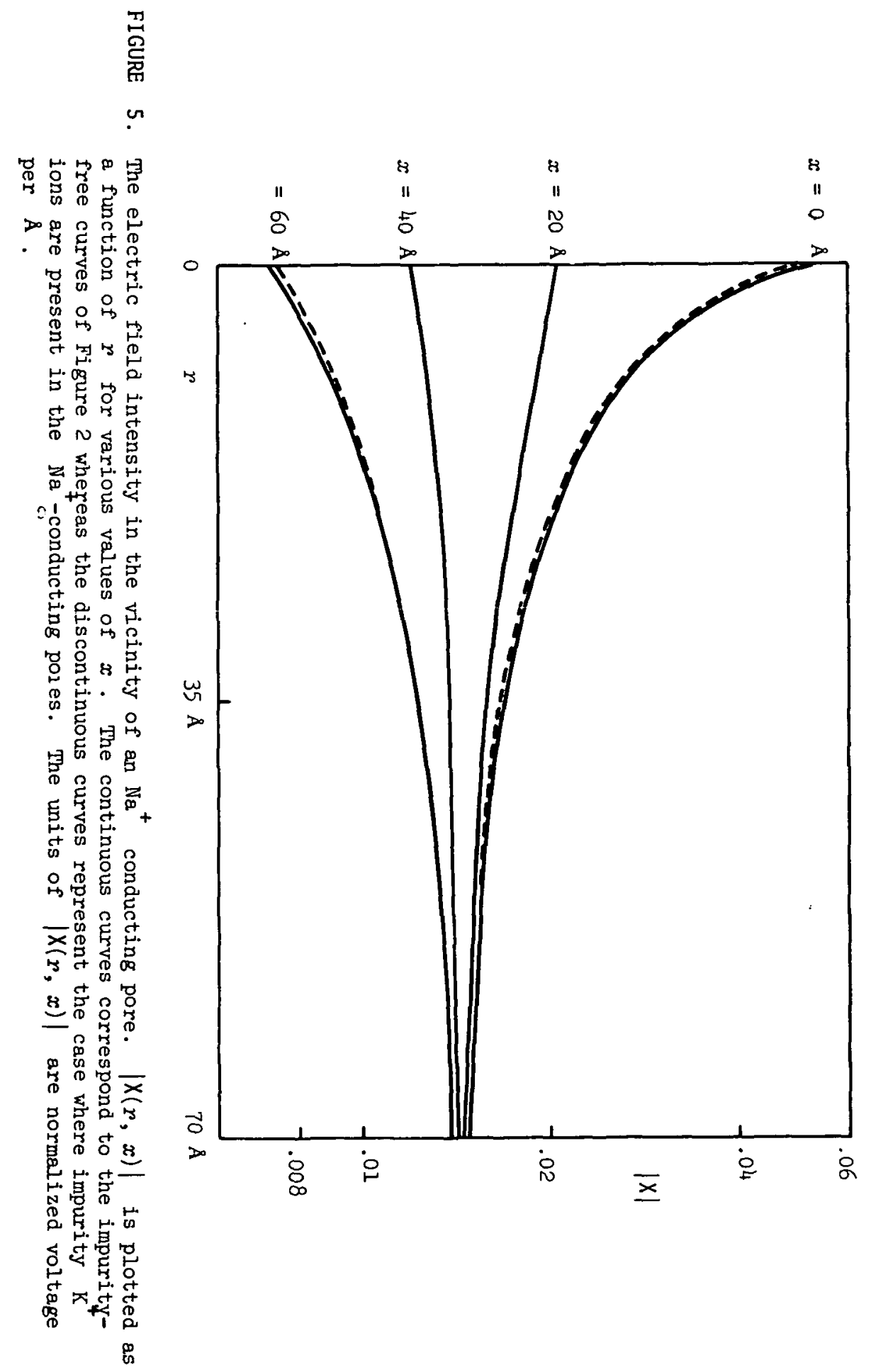


permeability control in the squid giant axonal pores, the corrected version of this section should be preferred.

Using the numerical techniques described in Section 5, the equipotentials and electric fields are computed in the vicinity of $j$-pores conducting impurity ions as well as $j$-ions: we employ the following values for the squid giant axon with axoplasm intracellularly and seawater extracellularly :

$$
\begin{aligned}
\pi_{\mathrm{K}} & =58.989, \text { which gives } \phi_{\mathrm{K}} \simeq 75 \mathrm{mV}, \\
\pi_{\mathrm{Na}} & =129.659, \text { which gives } \phi_{\mathrm{Na}} \simeq-52 \mathrm{mV} .
\end{aligned}
$$

This selection is made in order to have the $\phi_{j}$ agree with those quoted by Hodgkin and Huxley [12] at $5^{\circ} \mathrm{C}$. The $\Phi_{P j}(x)$ is given by (5.1) with the above values of $\left(s_{\delta} / S_{0}\right)_{j}$ replacing $\left(c_{\delta} / c_{0}\right)_{j}$ (and as in section 5 setting $\phi=1)$. The results of the calculation are shown in Figures 4 and 5 which show $|X(r, x)|$ for the impurity cases compared to that of the impurity-free cases of Section 5 .

\section{Ionic permeability}

We may evidently define ionic permeability $P_{j}$ of the transported species $j$ in terms of our model from (7.1)-(7.5) as

$$
P_{j}=e^{\prime} D_{j} \Omega_{j} / \delta
$$

thus isolating $P_{j}$ from the factors of $I_{j}$ which depend directly on extramembrane ionic concentration and on potential difference. The partition coefficient $\Omega_{j}$, assumed to be independent of $\phi$, must naturally be included as a factor of $P_{j}$ because the ion-selective characteristics of our model of facilitated transport play such a vital role in determining the properties of the conducting channels. If we assume that $\rho_{\mathrm{K}} / \rho_{\mathrm{Na}}$ remains constant for different axons then we have from Section 4 that $\Omega_{\mathrm{K}} \simeq \Omega_{\mathrm{Na}}$ for all axons. It follows that the classical description of the resting nerve, namely, that the membrane potential difference $\simeq \phi_{\mathrm{K}}$ because the permeability to $\mathrm{K}^{+}$is much greater than to 
$\mathrm{Na}^{+}$, implies from $(8.1)$ that $D_{\mathrm{K}} \gg D_{\mathrm{Na}}$ in the resting nerve.

Now determinations of the permeabilities of the squid giant axon by the use of radioactive isotopes of $\mathrm{Na}^{+}$and $\mathrm{K}^{+}$seem to contradict the classical view. For example, Tasaki [19] states that "the $\mathrm{Na}^{+}$ permeability of the resting axonal membrane was found to be roughly equal to $\mathrm{K}^{+}$permeability". Let us examine this conclusion in terms of our model. Denoting radioactive tracer currents and concentrations by an asterlsk we have from $(3.10)-(3.12)$ or $(7.1)-(7.5)$, for efflux $\left(c_{j}^{*}(\delta+)=0\right)$

$$
I_{j}^{*}=e^{\prime} D_{j} \Omega_{j} c_{j}^{*}(0-)\left(\phi / \phi_{j}-1\right) / \delta,
$$

and for influx $\left(c_{j}^{*}\left(0_{-}\right)=0\right)$

$$
I_{j}^{*}=-e^{\prime} D_{j} \Omega_{j} e_{j}^{*}(\delta+)\left(\phi / \phi_{j}-1\right) / \delta,
$$

with $\phi_{j}$ of course independent of tracer concentration. Now Tasaki [19] defines membrane permeability $P_{j T}$ as the radioactive tracer current density per unit original concentration of tracer which from (8.2) and (8.3) is

$$
P_{j T \text { efflux }}=e^{\prime} D_{j} \Omega_{j}\left(\phi / \phi_{j}-1\right) / \delta=P_{j T \text { influx }},
$$

as Tasaki indeed verifies, Furtḥermore since $\Omega_{\mathrm{K}} \simeq \Omega_{\mathrm{Na}},(8.4)$ implies that

$$
\frac{P_{\mathrm{Na} T}}{P_{\mathrm{KT}}}=\frac{D_{\mathrm{Na}}}{D_{\mathrm{K}}} \frac{\phi / \phi_{\mathrm{Na}}-1}{\phi / \phi_{\mathrm{K}}-1},
$$

and so we have for the natural squid giant axon, $P_{\mathrm{Na} T} / P_{\mathrm{K} T} \simeq 14 D_{\mathrm{Na}} / D_{\mathrm{K}}$ for the resting state. Tasaki and others actually find for axons treated with metabolic poison (to remove ambiguities which would otherwise arise from currents due to active transport) that $P_{\mathrm{NaT}} / P_{\mathrm{K} T} \simeq 1 / 3$ to unity which would make, on our model, $D_{\mathrm{Na}} / D_{\mathrm{K}}$ in the range $1 / 42$ to $1 / 14$. It follows from (8.1) that $P_{\mathrm{Na}} / P_{\mathrm{K}}$ lies in the same range of values. In other words, on our definition of the membrane permeability coefficients the radio-active tracer experiments aupport the classical view of the 
resting nerve.

\section{References}

[1] F. Brinley Jr., "Discussion report: 'Conference on newer properties perfused squid axons'", J. Gen. Physiol. 48 (1965), no. 5, 41-47.

[2] Kenneth S. Cole, "Electrodiffusion models for the membrane of squid giant axon", Physiol. Rev. 45 (1965), 340-379.

[3] Kenneth S. Cole, Membrane, ions and impulses: A chapter of classical biophysics (Biophysics Series, 1. University of California Press, Berkeley and Los Angeles, 1968).

[4] W.K. Chandler and H. Meves, "Sodium inactivation in internally perfused squid giant axons", Arch. Ges. Physiol. 281 (1964), 25-26.

[5] David E. Goldman, "Potential, impedance, and rectification in membranes", J. Gen. Physiol. 27 (1944), 37-60.

[6] Bertil Hille, "Pharmacological modifications of the sodium channels of frog nerve", J. Gen. Physiol. 51 (1968), 199-220.

[7] Bertil Hille, "The permeability of sodium channels to organic cations in myelinated nerve", J. Gen. Physiol. 58 (1971), 599-619.

[8] Bertil Hille, "Ionic channels in excitable membranes: current problems and biophysical approaches", Biophys. J. 22 (1978), 283-294.

[9] A.L. Hodgkin, The conduction of the nervous impulse (The Sherrington Lectures, 7. Liverpool University Press, Liverpool, 1964).

[10] A.L. Hodgkin and W.K. Chandler, "Effects of changes in ionic strength on inactivation and threshold in perfused nerve fibres of Loligo", J. Gen. Physiol. 48 (1965), no. 5, 27-30.

[11] A.L. Hodgkin and A.F. Huxley, "The components of membrane conductance in the giant axon of Loligo", J. Physiol. 116 (1952), 473-496. 
[12] A.L. Hodgkin and A.F. Huxley, "A quantitative description of membrane current and its application to conduction and excitation in nerve", J. Physiol. 117 (1952), 500-544.

[13] Bernard Katz, Nerve, muscle, and synapse (McGraw-Hill, New York, San Francisco, Sydney, 1966).

[14] Douglas Kenneth MclIroy, "A mathematical model of the nerve impulse at the molecular level", Math. Biosci. 7 (1970), 313-328.

[15] Douglas Kenneth Mcl!roy, "Analysis of the enzyme model of the nerve", Math. Biosci. 8 (1970), 109-129.

[16] D.K. Mcllroy and D.P. Mason, "Electric field dissociation of charged macromolecular sites in polarized biomembranes: III. Numerical results and application to nervous conduction", SIAM J. Appl. Math. 41 (1981), 580-591.

[17] T. Norahashl, "Dependence of resting and action potentials on internal potassium in perfused squid giant axons", J. Physiol. 169 (1963), 91-115.

[18] M. Planck, "Ueber die Erregung von Elektricät und Wärme in Elektrolyten", Wiedemann Ann. 39 (1890), 161-186.

[19] Ichiji Tasaki, "Permeability of squid axon membrane to various ions", J. Gen. Physiol. 46 (1963), 755-772.

[20] Ichiji Tasaki, Nerve excitation (Charles Thomas, Springfield, ]968).

Department of Applied Mathematics, University of the Witwatersrand, Johannesburg, South Africa. 